\title{
On the breaking of lepton flavor universality in $B$ decays
}

\author{
Admir Greljo, ${ }^{a, b}$ Gino Isidori ${ }^{a, c}$ and David Marzocca ${ }^{a}$ \\ ${ }^{a}$ Physik-Institut, Universität Zürich, \\ CH-8057 Zürich, Switzerland \\ ${ }^{b}$ Faculty of Science, University of Sarajevo, \\ Zmaja od Bosne 33-35, 71000 Sarajevo, Bosnia and Herzegovina \\ ${ }^{c}$ INFN - Laboratori Nazionali di Frascati, \\ I-00044 Frascati, Italy \\ E-mail: admir@physik.uzh.ch, isidori@physik.uzh.ch, \\ marzocca@physik.uzh.ch
}

AbstRACT: In view of recent experimental indications of violations of Lepton Flavor Universality (LFU) in $B$ decays, we analyze constraints and implications of LFU interactions, both using an effective theory approach, and an explicit dynamical model. We show that a simple dynamical model based on a $\mathrm{SU}(2)_{L}$ triplet of massive vector bosons, coupled predominantly to third generation fermions (both quarks and leptons), can significantly improve the description of present data. In particular, the model decreases the tension between data and SM predictions concerning: i) the breaking of $\tau$ - $\mu$ universality in $B \rightarrow D^{(*)} \ell \nu$ decays; ii) the breaking of $\mu-e$ universality in $B \rightarrow K \ell^{+} \ell^{-}$decays. Indirectly, the model might also decrease the discrepancy between exclusive and inclusive determinations of $\left|V_{c b}\right|$ and $\left|V_{u b}\right|$. The minimal version of the model is in tension with ATLAS and CMS direct searches for the new massive vectors (decaying into $\tau^{+} \tau^{-}$pairs), but this tension can be decreased with additional non-standard degrees of freedom. Further predictions of the model both at low- and high-energies, in view of future high-statistics data, are discussed.

Keywords: Beyond Standard Model, B-Physics

ARXiv EPrint: 1506.01705 


\section{Contents}

1 Introduction 1

2 The model 3

2.1 Step I: four-fermion operators 3

2.2 Step II: simplified dynamical model 4

3 Low-energy implications of the four-fermion operators 5

3.1 New physics effects in charged currents 5

3.2 Bounds from $\Delta F=2 \quad 6$

3.3 Bounds from LFU and LFV in $\tau$ decays, and neutrino physics $\quad 7$

3.4 New-physics effects in $b \rightarrow s \ell^{+} \ell^{-} \quad 8$

3.5 Combined fit and discussion 9

4 Constraints on the dynamical model $\quad 12$

$\begin{array}{lll}4.1 & \text { Bounds from LEP-I } & 12\end{array}$

$\begin{array}{ll}4.2 \text { High-energy searches } & 13\end{array}$

$\begin{array}{lll}5 & \text { Conclusions } & 17\end{array}$

\section{Introduction}

Recent experimental data in $B$ physics hint toward deviations of Lepton Flavor Universality (LFU) in semi-leptonic decays, both in the case of $b \rightarrow c$ charged-current transitions, as well as in the case of $b \rightarrow s$ neutral currents. The statistically most significant results can be summarized as follows:

- $3.8 \sigma+2.0 \sigma$ deviation of $\tau / \ell$ universality $(\ell=\mu, e)$ in $b \rightarrow c$ transitions, encoded by $[1-3]$ :

$$
\begin{aligned}
R_{D^{*}}^{\tau / \ell} & =\frac{\mathcal{B}\left(B \rightarrow D^{*} \tau \nu\right)_{\exp } / \mathcal{B}\left(B \rightarrow D^{*} \tau \nu\right)_{\mathrm{SM}}}{\mathcal{B}\left(B \rightarrow D^{*} \ell \nu\right)_{\exp } / \mathcal{B}\left(B \rightarrow D^{*} \ell \nu\right)_{\mathrm{SM}}}=1.28 \pm 0.08, \\
R_{D}^{\tau / \ell} & =\frac{\mathcal{B}(B \rightarrow D \tau \nu)_{\exp } / \mathcal{B}(B \rightarrow D \tau \nu)_{\mathrm{SM}}}{\mathcal{B}(B \rightarrow D \ell \nu)_{\exp } / \mathcal{B}(B \rightarrow D \ell \nu)_{\mathrm{SM}}}=1.37 \pm 0.18
\end{aligned}
$$

- $2.6 \sigma$ deviation of $\mu / e$ universality in $b \rightarrow s$ transitions [4]: ${ }^{1}$

$$
R_{K}^{\mu / e}=\left.\frac{\mathcal{B}\left(B \rightarrow K \mu^{+} \mu^{-}\right)_{\exp }}{\mathcal{B}\left(B \rightarrow K e^{+} e^{-}\right)_{\exp }}\right|_{q^{2} \in[1,6] \mathrm{GeV}}=0.745_{-0.074}^{+0.090} \pm 0.036 .
$$

\footnotetext{
${ }^{1}$ The result in eqs. (1.1) and (1.2) are obtained using $\mathcal{B}\left(B \rightarrow D^{*} \tau \nu\right) / \mathcal{B}\left(B \rightarrow D^{*} \ell \nu\right)_{\exp }=0.323 \pm 0.021$ and $\mathcal{B}(B \rightarrow D \tau \nu) / \mathcal{B}(B \rightarrow D \ell \nu)_{\exp }=0.41 \pm 0.05$ from the average of Babar [1], Belle [2], and LHCb [3], assuming $e / \mu$ universality in $b \rightarrow c \ell \nu$ decays, as indicated by $b \rightarrow c \ell \nu$ data [5] (see section 3.1), together with the theory predictions $\mathcal{B}\left(B \rightarrow D^{*} \tau \nu\right) / \mathcal{B}\left(B \rightarrow D^{*} \ell \nu\right)_{\mathrm{SM}}=0.252 \pm 0.003[6]$ and $\mathcal{B}(B \rightarrow D \tau \nu) / \mathcal{B}(B \rightarrow$ $D \ell \nu)_{\mathrm{SM}}=0.31 \pm 0.02[7]$. The $\mathrm{SM}$ expectation of $R_{K}^{\mu / e}$ is $\left|\left(R_{K}^{\mu / e}\right)_{\mathrm{SM}}-1\right|<1 \%$ [8] while, by construction, $R_{D^{*}}^{\tau / \ell}=R_{D}^{\tau / \ell}=1$ within the SM.
} 
In addition to these LFU ratios, whose deviation from unity would clearly signal physics beyond the Standard Model (SM), $B$-physics data exhibit other tensions with SM expectations in semi-leptonic observables. Most notably, a $\sim 3 \sigma$ deviation from the SM expectation has been reported by $\mathrm{LHCb}[9,10]$ in the so-called $P_{5}^{\prime}$ differential observable of $B \rightarrow K^{*} \mu^{+} \mu^{-}$decays [11]. Moreover, in charged current transitions there is a longstanding $\sim 2.5 \sigma$ discrepancy in the determination of both $\left|V_{c b}\right|$ and $\left|V_{u b}\right|$ from exclusive vs. inclusive semi-leptonic decays [12].

These deviations from the SM have triggered a series of theoretical speculations about possible New Physics (NP) interpretations, see in particular ref. [14-31]. Among these recent papers, two particularly interesting observations are: i) the proposal of ref. [20] to explain both $R_{K}^{\mu / e}$ and the $P_{5}^{\prime}$ anomaly by means of NP coupled dominantly to the third generation of quarks and leptons, with a small non-negligible mixing between third and second generations; ii) the observation of ref. [21] that is natural to establish a connection between $R_{K}^{\mu / e}$ and $R_{D^{*}}^{\tau / \ell}$ if the effective four-fermion semi-leptonic operators are build in terms of left-handed doublets.

Despite this recent progress, a coherent dynamical picture explaining all the anomalies has not emerged yet. On the one hand, a significantly improved fit of experimental data can be obtained with a specific set of four-fermion operators of the type $J_{q} \times J_{\ell}$, where $J_{q}$ and $J_{\ell}$ are flavor-non-universal left-handed quark and lepton currents [21,31]. On the other hand, even within an Effective Field Theory (EFT) approach, it is hard to believe that this set of effective operators is the only relevant one in explicit NP models. In particular, explicit NP models should face the tight constraints on four-quark and four-lepton operators dictated by meson-antimeson mixing, and by the bounds on Lepton Flavor Violation (LFV) and LF non-universality in pure leptonic processes. Moreover, the size of the SM modifications in eqs. (1.1)-(1.3) points toward relatively light new degrees of freedom, that could well be within the reach (or already excluded) by direct searches at the LHC.

In this paper we present an attempt to build a simplified coherent dynamical model able to explain, at least in part, these violations of LFU. The guiding principle of our construction is the idea that the $J_{q} \times J_{\ell}$ effective operators are generated by the exchange of one set (or more sets) of massive vector bosons that transform as a $\mathrm{SU}(2)_{L}$ triplet, and that are coupled to both quark and lepton currents. This hypothesis allows us to establish a connection between quark-lepton, quark-quark, and lepton-lepton effective operators. We further assume that the flavor structure of the new currents is consistent with an approximate $\mathrm{U}(2)_{q} \times \mathrm{U}(2)_{\ell}$ flavor symmetry acting on the first two generations of quarks and leptons, along the lines of ref. [32].

Under these assumptions we proceed with two main steps: i) we analyze the low-energy constraints (and the corresponding phenomenological implications) on the complete set of four-fermion operators generated within the model; ii) we discuss the additional constraints due to electroweak precisions test and collider searches, following from the specific choice of the mediators.

We find that, after taking into account all the existing constraints, the proposed model can still provide a significantly improved fit as far as low-energy observables are concerned. The most serious constraint on the model follows from the searches performed by ATLAS 
and CMS on new heavy neutral states $\left(Z^{\prime}\right)$ decaying into $\tau^{+} \tau^{-}$pairs. However, as we will discuss, the tension with direct searches can be decreased with additional non-standard degrees of freedom, whose net effect is the enhancement of the $Z^{\prime}$ decay width and the corresponding suppression of the $Z^{\prime} \rightarrow \tau^{+} \tau^{-}$branching ratio. The tension can be further reduced in the limit where the assumption of narrow resonances $(\Gamma \ll M)$, that is implicit in all present direct searches, no longer holds.

\section{The model}

\subsection{Step I: four-fermion operators}

Our main assumption is that all the non-standard four-fermion interactions can be described by the following effective Lagrangian

$$
\Delta \mathcal{L}_{4 f}^{(T)}=-\frac{1}{2 m_{V}^{2}} J_{\mu}^{a} J_{\mu}^{a}
$$

where $J_{\mu}^{a}$ is a fermion current transforming as a $\mathrm{SU}(2)_{L}$ triplet, built in terms of SM quarks and lepton fields:

$$
J_{\mu}^{a}=g_{q} \lambda_{i j}^{q}\left(\bar{Q}_{L}^{i} \gamma_{\mu} T^{a} Q_{L}^{j}\right)+g_{\ell} \lambda_{i j}^{\ell}\left(\bar{L}_{L}^{i} \gamma_{\mu} T^{a} L_{L}^{j}\right)
$$

Here $\lambda^{q, \ell}$ are Hermitian flavor matrices and, by convention, $\lambda_{33}^{q}=\lambda_{33}^{\ell}=1$.

We define $Q_{L}^{i}$ and $L_{L}^{i}$ to be the quark and lepton electroweak doublets in the flavor basis where down-type quarks and charged-leptons are diagonal. We assume an approximate $\mathrm{U}(2)_{q} \times \mathrm{U}(2)_{\ell}$ flavor symmetry, under which the light generations of $Q_{L}^{i}$ and $L_{L}^{i}$ transform as $2_{q} \times 1_{\ell}$ and $1_{q} \times 2_{\ell}$, respectively, and all other fermions are singlets. We further assume that the underlying dynamics responsible for the effective interaction in eq. (2.1) involves, in first approximation, only third generation SM fermions (the left-handed $1_{q} \times 1_{\ell}$ fermions). In this limit, the flavor couplings in eq. (2.2) are $\lambda_{i j}^{q, \ell}=\delta_{i 3} \delta_{3 j}$. The corrections to this limit are expected to be generated by appropriate $\mathrm{U}(2)_{q} \times \mathrm{U}(2)_{\ell}$ breaking spurions, connected to the generation of subleading terms in the Yukawa couplings for the SM light fermions.

In the quark case, the leading $\mathrm{U}(2)_{q}$ breaking spurion is a doublet, whose flavor structure is unambiguously connected to the CKM matrix $(V)$ [32]. We can thus expand $\lambda_{i j}^{q}$ as follows:

$$
\lambda_{i j}^{q}=\delta_{i 3} \delta_{3 j}+\left(\epsilon_{1} \delta_{i 3} \hat{V}_{3 j}+\epsilon_{1}^{*} \hat{V}_{3 i}^{*} \delta_{3 j}\right)+\epsilon_{2}\left(\hat{V}_{3 i}^{*} \hat{V}_{3 j}\right)+\ldots, \quad \hat{V}_{3 j}=V_{3 j}-\delta_{3 j} V_{3 j},
$$

with $\epsilon_{2}=O\left(\epsilon_{1}^{2}\right)$. As we will discuss below, low-energy flavor-physics data imply $\epsilon_{i} \ll 1$.

The breaking structure in the lepton sector is less clear, given the intrinsic ambiguity in reconstructing the lepton Yukawa couplings under the (natural) assumption that neutrino masses are generated by a see-saw mechanism. ${ }^{2}$ As we will discuss below, low-energy data are compatible with the hypothesis that the leading breaking terms in the lepton sector transform as doublets of $\mathrm{U}(2)_{\ell}$.

\footnotetext{
${ }^{2}$ An attempt to build a consistent neutrino mass matrix starting from an approximate $\mathrm{U}(2)_{\ell}$ symmetry broken by small $\mathrm{U}(2)_{\ell}$ doublets has been discussed in ref. [33].
} 
Among the four-fermion operators generated by the model, the ones most relevant to flavor phenomenology are:

$$
\begin{aligned}
\Delta \mathcal{L}_{\text {c.c. }}^{(T)} & =-\frac{g_{q} g_{\ell}}{2 m_{V}^{2}}\left[\left(V \lambda^{q}\right)_{i j} \lambda_{a b}^{\ell}\left(\bar{u}_{L}^{i} \gamma_{\mu} d_{L}^{j}\right)\left(\bar{\ell}_{L}^{a} \gamma_{\mu} \nu_{L}^{b}\right)+\text { h.c. }\right] \\
\Delta \mathcal{L}_{\mathrm{FCNC}}^{(T)} & =-\frac{g_{q} g_{\ell}}{4 m_{V}^{2}} \lambda_{a b}^{\ell}\left[\lambda_{i j}^{q}\left(\bar{d}_{L}^{i} \gamma_{\mu} d_{L}^{j}\right)-\left(V \lambda^{q} V^{\dagger}\right)_{i j}\left(\bar{u}_{L}^{i} \gamma_{\mu} u_{L}^{j}\right)\right]\left(\bar{\ell}_{L}^{a} \gamma_{\mu} \ell_{L}^{b}-\bar{\nu}_{L}^{a} \gamma_{\mu} \nu_{L}^{b}\right), \\
\Delta \mathcal{L}_{\Delta F=2}^{(T)} & =-\frac{g_{q}^{2}}{8 m_{V}^{2}}\left[\left(\lambda_{i j}^{q}\right)^{2}\left(\bar{d}_{L}^{i} \gamma_{\mu} d_{L}^{j}\right)^{2}+\left(V \lambda^{q} V^{\dagger}\right)_{i j}^{2}\left(\bar{u}_{L}^{i} \gamma_{\mu} u_{L}^{j}\right)^{2}\right] \\
\Delta \mathcal{L}_{\mathrm{LFV}}^{(T)} & =-\frac{g_{\ell}^{2}}{8 m_{V}^{2}} \lambda_{a b}^{\ell} \lambda_{c d}^{\ell}\left(\bar{\ell}_{L}^{a} \gamma_{\mu} \ell_{L}^{b}\right)\left(\bar{\ell}_{L}^{c} \gamma_{\mu} \ell_{L}^{d}\right) \\
\Delta \mathcal{L}_{\mathrm{LFU}}^{(T)} & =-\frac{g_{\ell}^{2}}{8 m_{V}^{2}}\left(-2 \lambda_{a b}^{\ell} \lambda_{c d}^{\ell}+4 \lambda_{a d}^{\ell} \lambda_{c b}^{\ell}\right)\left(\bar{\ell}_{L}^{a} \gamma_{\mu} \ell_{L}^{b}\right)\left(\bar{\nu}_{L}^{c} \gamma_{\mu} \nu_{L}^{d}\right) .
\end{aligned}
$$

\subsection{Step II: simplified dynamical model}

In order to generate $\Delta \mathcal{L}_{4 f}^{(T)}$ in a dynamical way, we introduce the heavy spin-1 triplet, $V_{\mu}^{a}$ $(a=1,2,3)$, following the general simplified Lagrangian proposed in ref. [45]. By means of this approach we can describe both models in which the new vector is weakly coupled, such as gauge extension of the SM, and strongly coupled models, such as Composite Higgs models. The simplified Lagrangian reads

$$
\mathcal{L}_{V}=-\frac{1}{4} D_{[\mu} V_{\nu]}^{a} D^{[\mu} V^{\nu] a}+\frac{m_{V}^{2}}{2} V_{\mu}^{a} V^{\mu a}+g_{H} V_{\mu}^{a}\left(H^{\dagger} T^{a} i \stackrel{\leftrightarrow}{D}{ }_{\mu} H\right)+V_{\mu}^{a} J_{\mu}^{a},
$$

where $T^{a}=\sigma^{a} / 2, D_{[\mu} V_{\nu]}^{a}=D_{\mu} V_{\nu}^{a}-D_{\nu} V_{\mu}^{a}$ and $D_{\mu} V_{\nu}^{a}=\partial_{\mu} V_{\nu}^{a}+g \epsilon^{a b c} W_{\mu}^{b} V_{\nu}^{c}{ }^{3}$

By integrating out at the tree-level the heavy spin-1 triplet and keeping only effective operators of dimension $\leq 6$, we obtain the effective Lagrangian

$$
\mathcal{L}_{\text {eff }}^{d=6}=-\frac{1}{2 m_{V}^{2}} J_{\mu}^{a} J_{\mu}^{a}-\frac{g_{H}^{2}}{2 m_{V}^{2}}\left(H^{\dagger} T^{a} i \stackrel{\leftrightarrow}{D} \mu H\right)\left(H^{\dagger} T^{a} i \stackrel{\leftrightarrow}{D}_{\mu} H\right)-\frac{g_{H}}{m_{V}^{2}}\left(H^{\dagger} T^{a} i \stackrel{\leftrightarrow}{D}_{\mu} H\right) J_{\mu}^{a}
$$

By construction, the first term is $\Delta \mathcal{L}_{4 f}^{(T)}$ in eq. (2.1). The second term, in the unitary gauge, is simply

$$
-\frac{g_{H}^{2} v^{2}}{4 m_{V}^{2}}\left(m_{W}^{2} W_{\mu}^{+} W_{\mu}^{-}+\frac{m_{Z}^{2}}{2} Z_{\mu} Z_{\mu}\right)\left(1+\frac{h}{v}\right)^{4} .
$$

This term induces an unphysical (custodially-invariant) shift in the $W$ - and $Z$-boson masses,. ${ }^{4}$ that can be reabsorbed by a redefinition of $v$, and deviations in the Higgs interactions to $W$ and $Z$ bosons. The latter are well within the existing bounds for the relevant set of parameters. The last term, instead, describes non-universal deviations in the $Z$ and $W$ couplings to SM quarks and leptons that lead to non-trivial constraints on the parameter space of the model (see section 4.1).

\footnotetext{
${ }^{3}$ With respect to ref. [45] we dropped interaction terms with two or more insertions of $V_{\mu}^{a}$. While such terms can be relevant for double production, they do not contribute to the low-energy effective Lagrangian at the dimension- 6 level and are thus largely unconstrained by low-energy data.

${ }^{4}$ Within the full model of eq. (2.9) this corresponds to a mass mixing between the SM EW gauge bosons and the heavy vector triplet. The relative shift in the heavy vector masses $m_{V}$ is only of $O\left(g_{H}^{2} m_{W}^{2} v^{2} / m_{V}^{4}\right)$
} 


\section{Low-energy implications of the four-fermion operators}

\subsection{New physics effects in charged currents}

Since the new interactions are purely left-handed, in the case of charged currents their effect is simply an overall (flavor non-universal) rescaling of the SM amplitudes:

$$
R_{\ell^{i}}^{b \rightarrow c}=\frac{\mathcal{A}\left(b \rightarrow c \ell^{i} \bar{\nu}^{i}\right)_{\mathrm{SM}+\mathrm{NP}}}{\mathcal{A}\left(b \rightarrow c \ell^{i} \bar{\nu}^{i}\right)_{\mathrm{SM}}}=1+R_{0} \lambda_{i i}^{\ell}\left(1+\frac{V_{c s}\left(\lambda_{b s}^{q}\right)^{*}+V_{c d}\left(\lambda_{b s}^{q}\right)^{*}}{V_{c b}}\right),
$$

where

$$
R_{0}=\frac{g_{q} g_{\ell} m_{W}^{2}}{g^{2} m_{V}^{2}} \equiv \frac{G_{F}^{(T)}}{G_{F}^{\mathrm{SM}}} .
$$

Using this expression, the LFU breaking ratio in eq. (1.1) assumes the form

$$
R_{D^{*}}^{\tau / \ell} \approx 1+2 R_{0} \operatorname{Re}\left[\left(1-\frac{\lambda_{\mu \mu}^{\ell}+\lambda_{e e}^{\ell}}{2}\right)\left(1+\frac{V_{c s}\left(\lambda_{b s}^{q}\right)^{*}+V_{c d}\left(\lambda_{b d}^{q}\right)^{*}}{V_{c b}}\right)\right] \approx 1+2 R_{0},
$$

where we have assumed $\left|\lambda_{\mu \mu, e e}^{\ell}\right| \ll 1$ and $\left|\lambda_{i j}^{q}\right| \ll\left|V_{3 i}^{*} V_{3 j}\right|$. The first condition is required by the smallness of deviations from the SM in $b \rightarrow c \ell \nu$ decays (see below), the second condition follows by the consistency of the bounds from $\Delta F=2$ amplitudes (see section 3.2). We are thus able to fix the overall strength of the new effective charged-current interaction (compared to the Fermi coupling):

$$
R_{0}=\frac{1}{2}\left(R_{D^{*}}^{\tau / \ell}-1\right)=0.14 \pm 0.04
$$

The model predicts the same violation of $\tau / \ell$ universality for all type of $b \rightarrow c$ and $b \rightarrow u$ transitions. This implies, in particular, $R_{D}^{\tau / \ell}=R_{D^{*}}^{\tau / \ell}$, that is perfectly consistent with the experimental result in eq. (1.2).

In principle, violations of LFU universality are expected also between $b \rightarrow c(u) \mu \nu$ and $b \rightarrow c(u) e \nu$ modes. Unfortunately, it is very difficult to get experimental bounds on the latter using published data, since most of the high-statistics semi-leptonic analyses are performed combining $\mu$ and $e$ modes [5]. Using the PDG fit for the combined $B^{ \pm} / B^{0}$ sample [5], that is separated for $\mu$ and $e$ modes, we deduce that deviations between $\Gamma(b \rightarrow$ $c(u) \mu \nu)$ and $\Gamma(b \rightarrow c(u) e \nu)$ as large as $\sim 2 \%$ are allowed by present data. Within our model, we expect

$$
\frac{\Gamma(b \rightarrow c(u) \mu \bar{\nu})_{\mathrm{SM}+\mathrm{NP}}}{\Gamma(b \rightarrow c(u) e \bar{\nu})_{\mathrm{SM}+\mathrm{NP}}} \approx 1+2 R_{0}\left(\lambda_{\mu \mu}^{\ell}-\lambda_{e e}^{\ell}\right)
$$

The strong constraints on LFU involving only quarks and leptons of the first two generations ( $\pi$ and $K$ decays, CKM unitarity, and $\mu$ decay [34]) implies $\left|\lambda_{e e}^{\ell}\right| \ll\left|\lambda_{\mu \mu}^{\ell}\right|$. As a result, the constraints on $\mu-e$ charged-current LFU can be used to set the approximate bound

$$
\left|\lambda_{\mu \mu}^{\ell}\right| \lesssim 0.07\left(\frac{0.15}{R_{0}}\right),
$$


that justifies having neglected $\lambda_{\mu \mu}^{\ell}$ in eq. (3.3). After this bound is imposed, violations of LFU universality in $K$ and $\pi$ semileptonic decays turn out to be unobservables, given the additional suppression factor $\left|V_{u b}\left(\lambda_{b q}^{q}\right)^{*} / V_{u q}\right|$, for $q=s, d$, compared to eq. (3.5).

The universal $30 \%$ excess to $\tau$ semi-leptonic charged-current decays is likely to explain, at least in part, the tension between exclusive and inclusive determinations of $\left|V_{c b}\right|$ and $\left|V_{u b}\right|$. The argument goes as follows: the $B \rightarrow X_{c, u} \tau \nu$ decays followed by $\tau \rightarrow X \ell \nu \nu$ represent a background for the inclusive $B \rightarrow X_{c, u} \ell \nu$ analyses. At present, this background is subtracted via montecarlo simulations that assume a SM-like $\mathcal{B}\left(B \rightarrow X_{c, u} \tau \nu\right) .{ }^{5}$ This procedure therefore underestimates the background events and leads to an enhanced $B \rightarrow$ $X_{c, u} \ell \nu$ signal. On the other hand, the problem is not present in the exclusive decays of the type $B \rightarrow D^{(*)} \ell \nu$, where the kinematical closure of the events prevents the contamination from $\tau$ decays. A precise estimate of the effect would require a re-analysis of $B \rightarrow X_{c, u} \ell \nu$ data and is beyond the scope of the present paper. However, we note that this effect necessarily goes in the right direction (enhanced signal in inclusive modes), and that is likely to be larger in $b \rightarrow u$ compared to $b \rightarrow c$, given the different kinematical cuts. We are then led to the conclusion that the most reliable estimates of $\left|V_{c b}\right|$ and $\left|V_{u b}\right|$ are those obtained by means of exclusive decays and, more specifically, exclusive decays into electron final states.

As a result of this discussion, we urge the experimental collaborations to reanalyze all semi-leptonic charged-current $B$ decays without imposing LFU, both as far as signal and as far as background are concerned.

\subsection{Bounds from $\Delta F=2$}

Also in the case of $\Delta F=2$ transitions the new interaction amounts to an overall flavor non-universal rescaling of the SM amplitudes. It is therefore convenient to define the ratios

$$
R_{B_{q}}^{\Delta F=2}=\frac{\mathcal{A}\left(B_{q} \rightarrow \bar{B}_{q}\right)_{\mathrm{SM}+\mathrm{NP}}}{\mathcal{A}\left(B_{q} \rightarrow \bar{B}_{q}\right)_{\mathrm{SM}}}=1+R_{0} \frac{g_{q}}{g_{\ell}} \frac{\left(\lambda_{b q}^{q}\right)^{2}}{\left(V_{t b}^{*} V_{t q}\right)^{2}} \times\left(R_{\mathrm{SM}}^{\text {loop }}\right)^{-1}
$$

where $^{6}$

$$
R_{\mathrm{SM}}^{\mathrm{loop}}=\frac{\alpha_{\mathrm{em}} S_{0}\left(x_{t}\right)}{4 \pi s_{W}^{2}} \approx 6.5 \times 10^{-3} .
$$

The consistency with experimental results on down-type $\Delta F=2$ amplitudes, where no significant deviations from the SM are observed (up to the 10\%-30\% level depending on the specific amplitude) implies $\left|\lambda_{i j}^{q}\right| \lesssim 10^{-1}\left|V_{3 i}^{*} V_{3 j}\right|$, for $R_{0}=0.15$ and $g_{\ell} / g_{q}=O(1)$. As anticipated, this justifies the expansion on the r.h.s. of eq. (3.3).

If the corrections of $\lambda_{i j}^{q}$ from the leading term are generated by $\mathrm{U}(2)_{q}$ breaking spurions, as proposed in eq. (2.3), the $R_{B_{q}}^{\Delta F=2}$ terms should respect the $\mathrm{U}(2)^{3}$ prediction [32]

$$
R_{B_{s}}^{\Delta F=2}=R_{B_{d}}^{\Delta F=2}
$$

\footnotetext{
${ }^{5}$ We thank Nicola Serra for clarifications about this point.

${ }^{6}$ For the SM amplitude and the definition for the loop function $S_{0}\left(x_{t}\right)=S_{0}\left(m_{t}^{2} / m_{W}^{2}\right) \approx 2.4$ see e.g. ref. [37].
} 
that, in turn, implies no corrections to the clean ratio $\Delta M_{B_{s}} / \Delta M_{B_{d}}$. Furthermore, if the $\epsilon_{1}$ parameter in the expansion (2.3) is real, we expect no corrections to the $C P$-violating phases of $B_{s}$ and $B_{d}$ mixing.

To discuss the NP impact in $b \rightarrow s \ell^{+} \ell^{-}$decays we need to establish a precise upper bound on $\left|\lambda_{b s}^{q}\right|$. According to the $\mathrm{U}(2)^{3}$ fit of meson-antimeson mixing of ref. [36], we set $R_{B_{s}}^{\Delta F=2} \in[0.8,1.2]$ at $95 \% \mathrm{CL}$, that implies

$$
\left|\lambda_{b s}^{q}\right|<\left|\lambda_{b s}^{q}\right| \max =0.093\left|V_{t s}\right|\left|\frac{g_{\ell}}{g_{q}}\right|^{1 / 2}\left(\frac{0.15}{R_{0}}\right)^{1 / 2} .
$$

Even in the limit of negligible $\lambda_{i j}^{q}$ for $i \neq 3$ or $j \neq 3$, a potentially sizable contribution to $\Delta C=2$ is generated by CKM mixing, starting from the leading term in eq. (2.3). This can be written as

$$
\begin{aligned}
\Delta \mathcal{L}_{\Delta C=2}^{(V)} & =-\frac{1}{\Lambda_{u c}^{2}} \frac{\left(V_{u b} V_{c b}^{*}\right)^{2}}{\left|V_{u b} V_{c b}^{*}\right|^{2}}\left(\bar{u}_{L} \gamma_{\mu} c_{L}\right)^{2}+\text { h.c. } \\
\Lambda_{u c} & =\left[\frac{G_{F}}{\sqrt{2}} R_{0} \frac{g_{q}}{g_{\ell}}\left|V_{u b} V_{c b}^{*}\right|^{2}\right]^{-1 / 2} \approx 6.9 \times 10^{3} \mathrm{TeV} \times\left|\frac{g_{\ell}}{g_{q}}\right|^{1 / 2}\left(\frac{0.15}{R_{0}}\right)^{1 / 2} .
\end{aligned}
$$

Remarkably, for $g_{\ell} / g_{q}=O(1)$ this result is compatible with the existing bounds from CP violation in $D-\bar{D}$ mixing that require $\Lambda_{u c}>3 \times 10^{3} \mathrm{TeV}$ [38]. For $R_{0}=0.15$ this fixes $\left|\frac{g_{q}}{g_{\ell}}\right| \lesssim 5.4$

\subsection{Bounds from LFU and LFV in $\tau$ decays, and neutrino physics}

LFU in $\tau$ decays has been tested at the permil level. Assuming $\lambda_{i j}^{\ell}$ is negligible if $i=e$ or $j=e$, and imposing $\left|\lambda_{i j}^{q}\right| \ll\left|V_{3 i}^{*} V_{3 j}\right|$, such tests can be used to set stringent limits on $\left|\lambda_{\mu \mu}^{\ell}\right|$ and $\left|\lambda_{\tau \mu}^{\ell}\right|$. Moreover, a strong limit on the product $\left|\lambda_{\tau \mu}^{\ell}\right|\left|\lambda_{\mu \mu}^{\ell}\right|$ follows from the upper bound on $\mathcal{B}(\tau \rightarrow 3 \mu)$.

The relevant modified effective Lagrangians are

$$
\begin{aligned}
& \Delta \mathcal{L}_{\mathrm{LFU}}^{(T)}=-\frac{g_{\ell}^{2}}{2 m_{V}^{2}}\left[\left(\lambda_{\mu \mu}^{\ell}-\frac{1}{2}\left|\lambda_{\tau \mu}^{\ell}\right|^{2}\right)\left(\bar{\tau}_{L} \gamma_{\mu} \mu_{L}\right)\left(\bar{\nu}_{\mu} \gamma_{\mu} \nu_{\tau}\right)+\frac{1}{2} \lambda_{\tau \mu}^{\ell}\left(\bar{\tau}_{L} \gamma_{\mu} \mu_{L}\right)\left(\bar{\nu}_{\tau} \gamma_{\mu} \nu_{\tau}\right)+\text { h.c. }\right], \\
& \Delta \mathcal{L}_{\mathrm{LFV}}^{(T)}=-\frac{1}{\Lambda_{\tau \mu}^{2}}\left(\bar{\tau}_{L} \gamma_{\mu} \mu_{L}\right)\left(\bar{\mu}_{L} \gamma_{\mu} \mu_{L}\right), \quad \Lambda_{\tau \mu}=\left[\frac{G_{F}}{\sqrt{2}} R_{0} \frac{g_{\ell}}{g_{q}} \lambda_{\mu \mu}^{\ell} \lambda_{\tau \mu}^{\ell}\right]^{-1 / 2} .
\end{aligned}
$$

As far as LFU tests are concerned, the observable we consider is [39]

$$
\frac{\mathcal{B}(\tau \rightarrow \mu \bar{\nu} \nu) f\left(x_{e}^{2}\right)}{\mathcal{B}(\tau \rightarrow e \bar{\nu} \nu) f\left(x_{\mu}^{2}\right)}=\left|1+R_{0} \frac{g_{\ell}}{g_{q}}\left(\lambda_{\mu \mu}^{\ell}-\frac{1}{2}\left|\lambda_{\tau \mu}^{\ell}\right|^{2}\right)\right|^{2}+\left|\frac{R_{0}}{2} \frac{g_{\ell}}{g_{q}} \lambda_{\tau \mu}^{\ell}\right|^{2}=1.0040 \pm 0.0032,
$$

where $x_{\ell}=\frac{m_{\ell}}{m_{\tau}}, f\left(x_{\mu}^{2}\right) / f\left(x_{e}^{2}\right)=0.9726$ is a phase space factor, and we summed over neutrinos of arbitrary flavor. The numerical result on the r.h.s. of eq. (3.14) is obtained using PDG data [5]. Expanding to first order in $R_{0}$ and assuming $\left|\lambda_{\tau \mu}^{\ell}\right|^{2} \ll\left|\lambda_{\mu \mu}^{\ell}\right|$ we obtain

$$
\lambda_{\mu \mu}^{\ell}=(0.013 \pm 0.011) \times \frac{g_{q}}{g_{\ell}}\left(\frac{0.15}{R_{0}}\right)
$$


This constraint is significantly stronger than the bound from $b \rightarrow c \mu(e) \nu$ universality (eq. (3.6)), unless $g_{q} / g_{\ell} \gg 1$.

In principle, an independent bound on $\left|\lambda_{\mu \mu}^{\ell}\right|$ can be obtained by neutrino trident production, namely muon pair production from $\nu_{\mu}$ scattering on a heavy nuclei. The inclusive cross section $\sigma\left(\nu_{\mu} N \rightarrow \nu_{\mu} N \mu^{+} \mu^{-}\right)$is proportional to the combination of effective couplings $\left(C_{V}^{2}+C_{A}^{2}\right)$ where $C_{V, A}=C_{V, A}^{\mathrm{SM}}+\Delta C_{V, A}$ [40], and the SM reference values are $C_{V}^{\mathrm{SM}}=1 / 2+2 \sin ^{2} \theta_{W}$ and $C_{A}^{\mathrm{SM}}=1 / 2$ [40]. The corrections to these couplings in our model are

$$
\Delta C_{V}=\Delta C_{A}=\frac{m_{W}^{2} g_{\ell}^{2}}{2 m_{V}^{2} g^{2}}\left|\lambda_{\mu \mu}^{\ell}\right|^{2} .
$$

Combining the reported cross section measurements from the CHARM-II collaboration $\left(\sigma / \sigma^{\mathrm{SM}}=1.58 \pm 0.57[41]\right)$ and the CCFR collaboration $\left(\sigma / \sigma^{\mathrm{SM}}=0.82 \pm 0.28[42]\right)$, we find

$$
\left|\lambda_{\mu \mu}^{\ell}\right|<1.5\left|\frac{g_{q}}{g_{\ell}}\right|^{1 / 2}\left(\frac{0.15}{R_{0}}\right)^{1 / 2}
$$

that is well below the LFU bound in eq. (3.15).

As far as LFV is concerned, the $\mathcal{B}(\tau \rightarrow 3 \mu)<2.1 \times 10^{-8}$ bound [5] implies $\Lambda_{\tau \mu}>$ $11 \mathrm{TeV}$, that can be translated into

$$
\left|\lambda_{\mu \mu}^{\ell} \lambda_{\tau \mu}^{\ell}\right|<0.005\left|\frac{g_{q}}{g_{\ell}}\right|\left(\frac{0.15}{R_{0}}\right) .
$$

If $\left|\lambda_{\mu \mu}^{\ell}\right|$ assumes the maximal allowed by eq. (3.15), we are left with the bound $\left|\lambda_{\tau \mu}^{\ell}\right| \lesssim 0.15$. The latter is compatible with the hypothesis that $\lambda_{i j}^{\ell}$ admits an expansion similar to that of $\lambda_{i j}^{q}$ in eq. (2.3), or that the leading breaking of the $\mathrm{U}(2)_{\ell}$ flavor symmetry is determined by spurions transforming as $\mathrm{U}(2)_{\ell}$ doublets.

\subsection{New-physics effects in $b \rightarrow s \ell^{+} \ell^{-}$}

The effective Lagrangian encoding NP effects in $b \rightarrow s \ell^{+} \ell^{-}(\ell=e, \mu, \tau)$ is

$$
\Delta \mathcal{L}_{b \rightarrow s \ell^{+} \ell^{-}}^{(V)}=-\frac{2 G_{F}}{\sqrt{2}} R_{0} \lambda_{b s}^{q}\left(\bar{b}_{L} \gamma_{\mu} s_{L}\right)\left(\bar{\tau}_{L} \gamma_{\mu} \tau_{L}+\lambda_{\mu \mu}^{\ell} \bar{\mu}_{L} \gamma_{\mu} \mu_{L}+\lambda_{e e}^{\ell} \bar{e}_{L} \gamma_{\mu} e_{L}\right)
$$

Using $\Delta \mathcal{L}_{b \rightarrow s \ell^{+} \ell^{-}}^{(V)}$ to determine modified matching conditions for the Wilson coefficients of the most general $b \rightarrow s \ell^{+} \ell^{-}$effective Hamiltonian,

$$
\mathcal{H}_{\mathrm{eff}}^{b \rightarrow s}=-\frac{4 G_{F}}{\sqrt{2}} V_{t b} V_{t s}^{*} \frac{e^{2}}{16 \pi^{2}} \sum_{i}\left(C_{i}^{\ell} O_{i}^{\ell}+C_{i}^{\ell \prime} O^{\ell \prime}\right)+\text { h.c. }
$$

leads to

$$
\Delta C_{9}^{\tau}=-\Delta C_{10}^{\tau}=-\frac{\pi R_{0}}{\alpha_{\mathrm{em}}} \frac{\lambda_{b s}^{q}}{V_{t b}^{*} V_{t s}}, \quad \Delta C_{9}^{\mu(e)}=-\Delta C_{10}^{\mu(e)}=-\lambda_{\mu \mu(e e)}^{\ell} \Delta C_{10}^{\tau},
$$

where

$$
O_{9}^{\ell}=\left(\bar{s}_{L} \gamma^{\nu} b_{L}\right) \bar{\ell} \gamma_{\nu} \ell, \quad O_{10}^{\ell}=\left(\bar{s}_{L} \gamma^{\nu} b_{L}\right) \bar{\ell} \gamma_{\nu} \gamma^{5} \ell
$$


Present $b \rightarrow s \mu^{+} \mu^{-}$anomalies and $R_{K}^{\mu / e}$ seems to indicate a LF non-universal modification in the Wilson coefficients $C_{9}^{\mu}$ compared to the SM (see e.g. ref. [14, 15]). However, a good fit to present data is also obtained assuming $\Delta C_{9}^{\mu}=-\Delta C_{10}^{\mu} \neq 0$ and $\Delta C_{9,10}^{e}=0$, that is compatible with the modification expected in our NP framework for $\left|\lambda_{\mu \mu}^{\ell}\right| \gg\left|\lambda_{e e}^{\ell}\right|$. The best fit thus obtained implies $\Delta C_{9}^{\mu}=-\Delta C_{10}^{\mu}=-0.53 \pm 0.18[43,44]$.

In order to reproduce this result within our model we must impose

$$
\lambda_{b s}^{q} \lambda_{\mu \mu}^{\ell}=(3.4 \pm 1.1) \times 10^{-4} \times\left(\frac{0.15}{R_{0}}\right) .
$$

This result is in some tension with the bounds on $\left|\lambda_{\mu \mu}^{\ell}\right|$ and $\left|\lambda_{b s}^{q}\right|$ dictated by LFU in $\tau$ decays and $\Delta m_{B_{q}}$ mixing, respectively. To express this tension more clearly, it is convenient to normalize eq. (3.23) to the maximal value of $\left|\lambda_{b s}^{q}\right|$ allowed by $\Delta m_{B_{q}}$ mixing. This leads to

$$
\frac{\lambda_{b s}^{q}}{\left|\lambda_{b s}^{q}\right| \max }\left(\frac{R_{0}}{0.15}\right)^{1 / 2}\left|\frac{g_{\ell}}{g_{q}}\right|^{1 / 2} \lambda_{\mu \mu}^{\ell}=(0.09 \pm 0.03),
$$

that should be compared with the constraint on $\left|\lambda_{\mu \mu}^{\ell}\right|$ from eq. (3.15). Given the different scaling of eq. (3.24) and eq. (3.15) in terms of $g_{\ell} / g_{q}$, the tension decreases for $\left|g_{\ell} / g_{q}\right|<1$.

As far as $b \rightarrow s \tau^{+} \tau^{-}$decays are concerned, for $R_{0}=0.15$ and $g_{q}=g_{\ell}$, we find

$$
\Delta C_{9}^{\tau}=-\Delta C_{10}^{\tau} \approx-5.6 \times \frac{\lambda_{b s}^{q}}{\left|\lambda_{b s}^{q}\right|_{\max }}, \quad \text { vs. } \quad\left(C_{9}^{\tau}\right)_{\mathrm{SM}} \approx-\left(C_{10}^{\tau}\right)_{\mathrm{SM}} \approx 4.2 .
$$

Thus if $\lambda_{b s}^{q}$ is close to $\left|\lambda_{b s}^{q}\right|_{\max }$, as favored by $b \rightarrow s \mu^{+} \mu^{-}$anomalies and $R_{K}^{\mu / e}$, depending on $\arg \left(\lambda_{b s}^{q}\right)$ we have two very different non-standard predictions for $b \rightarrow s \tau^{+} \tau^{-}$decays. In the case of maximal constructive interference of NP and SM amplitudes, $b \rightarrow s \tau^{+} \tau^{-}$ rates could be enhanced up to a factor $\approx 5$ over the SM; in the case of maximal destructive interference, $b \rightarrow s \tau^{+} \tau^{-}$rates could be strongly suppressed (even less than 1/10) compared to the SM expectation. This possible enhancement or suppression would hold also for the $b \rightarrow s \nu_{\tau} \bar{\nu}_{\tau}$ rates, but it would appear "diluted" by a factor of $\approx 3$ in the measurable $b \rightarrow s \nu \bar{\nu}$ rates summed over all neutrino species.

In principle, the effective four-fermion Lagrangian in eq. (2.1) could allow also FCNCLFV transitions of the type $b \rightarrow s \ell_{i}^{ \pm} \ell_{j}^{\mp}$, with the largest amplitude expected for $b \rightarrow s \tau^{ \pm} \mu^{\mp}$. The latter can be estimated by means of eq. (3.21), with the replacement $\lambda_{\mu \mu}^{\ell} \rightarrow \lambda_{\tau \mu}^{\ell}$. Given the constraint on $\left|\lambda_{\tau \mu}^{\ell}\right|$ in eq. (3.18), we find that FCNC-LFV helicity-conserving transitions $\left(B \rightarrow K \tau^{ \pm} \mu^{\mp}, B \rightarrow K^{*} \tau^{ \pm} \mu^{\mp}, \ldots\right)$ can have rates which are at most $10 \%$ of those of the corresponding di-muon modes in the SM. Similarly, we find $\mathcal{B}\left(B_{s} \rightarrow \tau^{ \pm} \mu^{\mp}\right) \lesssim 10^{-8}$. These bounds makes the experimental search of these FCNC-LFV transitions very challenging, at least in the short term. We also note that such bounds are saturated only if $\mathcal{B}(\tau \rightarrow 3 \mu)$ is just below its current experimental bound.

\subsection{Combined fit and discussion}

The low-energy observables discussed above depend on the three flavor-non-universal couplings $\lambda_{b s}^{q}, \lambda_{\mu \mu}^{\ell}, \lambda_{\tau \mu}^{\ell}$, and the two flavor-independent combinations

$$
\epsilon_{\ell, q} \equiv \frac{g_{\ell, q} m_{W}}{g m_{V}} \approx g_{\ell, q} \frac{122 \mathrm{GeV}}{m_{V}},
$$




\begin{tabular}{|c|c|c|}
\hline Obs. $\mathcal{O}_{i}$ & Exp. bound $\left(\mu_{i} \pm \sigma_{i}\right)$ & Def. $\mathcal{O}_{i}\left(x_{\alpha}\right)$ \\
\hline$R_{0}\left(D^{*}\right)$ & $0.14 \pm 0.04$ & $\epsilon_{\ell} \epsilon_{q}$ \\
$R_{0}(D)$ & $0.19 \pm 0.09$ & $\epsilon_{\ell} \epsilon_{q}$ \\
$\Delta R_{b \rightarrow c}^{\mu e}$ & $0.00 \pm 0.01$ & $2 \epsilon_{\ell} \epsilon_{q} \lambda_{\mu \mu}^{\ell}$ \\
$\Delta R_{B_{s}}^{\Delta F=2}$ & $0.0 \pm 0.1$ & $\epsilon_{q}^{2}\left|\lambda_{b s}^{q}\right|^{2}\left(\left|V_{t b}^{*} V_{t s}\right|^{2} R_{\mathrm{SM}}^{\mathrm{loop}}\right)^{-1}$ \\
$\Delta C_{9}^{\mu}$ & $-0.53 \pm 0.18$ & $-\left(\pi / \alpha_{\mathrm{em}}\right) \lambda_{\mu \mu}^{\ell} \epsilon_{\ell} \epsilon_{q} \lambda_{b s}^{q} /\left|V_{t b}^{*} V_{t s}\right|$ \\
$\Delta R_{\tau \rightarrow \mu / e}$ & $0.0040 \pm 0.0032$ & $2 \epsilon_{\ell}^{2}\left(\lambda_{\mu \mu}^{\ell}-\frac{1}{2}\left|\lambda_{\tau \mu}^{\ell}\right|^{2}\right)$ \\
$\Lambda_{\tau \mu}^{-2}$ & $(0.0 \pm 4.1) \times 10^{-9}\left[\mathrm{GeV}^{-2}\right]$ & $\left(G_{F} / \sqrt{2}\right) \epsilon_{\ell}^{2} \lambda_{\mu \mu}^{\ell} \lambda_{\tau \mu}^{\ell}$ \\
$\Lambda_{u c}^{-2}$ & $(0.0 \pm 5.6) \times 10^{-14}\left[\mathrm{GeV}^{-2}\right]$ & $\left(G_{F} / \sqrt{2}\right) \epsilon_{q}^{2}\left|V_{u b} V_{c b}^{*}\right|^{2}$ \\
\hline
\end{tabular}

Table 1. Observables entering in the fit with their experimental bound (assuming the uncertainties follow the Gaussian distribution) and the expression in terms of the parameters of our model.

which we assume to be bounded by $\left|\epsilon_{\ell, q}\right|<2$. We have performed a combined fit of these parameters using the experimental constraints reported in table 1. For simplicity, we have assumed Gaussian errors for all the observables. The preferred region of the model parameters $\left(x_{\alpha}\right)$ has been determined minimizing the $\chi^{2}$ distribution

$$
\chi^{2}\left(x_{\alpha}\right)=\sum_{i} \frac{\left(\mathcal{O}_{i}\left(x_{\alpha}\right)-\mu_{i}\right)^{2}}{\sigma_{i}^{2}} .
$$

The best-fit point is found for

$$
\epsilon_{\ell} \approx 0.37, \quad \epsilon_{q} \approx 0.38, \quad \lambda_{b s}^{q} \approx 2.3 \times 10^{-3}, \quad \lambda_{\mu \mu}^{\ell} \approx 2.0 \times 10^{-2}, \quad \lambda_{\tau \mu}^{\ell} \approx 4.8 \times 10^{-2} .
$$

The $\chi^{2}$ improvement of the best-fit point with respect to the SM limit is $\chi^{2}\left(x_{\mathrm{SM}}\right)-$ $\chi^{2}\left(x_{\mathrm{BF}}\right)=18.6$ for 5 d.o.f., which corresponds to a $p$-value for the SM hypothesis of 0.002 . In figure 1 we show the $68 \% \mathrm{CL}$ and $95 \% \mathrm{CL}$ regions in the $\left(\epsilon_{q}, \epsilon_{\ell}\right),\left(\lambda_{b s}^{q}, \lambda_{\mu \mu}^{\ell}\right),\left(\lambda_{\mu \mu}^{\ell}, \lambda_{\tau \mu}^{\ell}\right)$, and $\left(\Delta C_{9}^{\mu}, \Delta R_{B_{s}}\right)$ planes, after having marginalised over the other parameters.

The best-fit point implies a small non-standard contribution to $C_{9}^{\mu}$. This is because of the bounds on $\left|\lambda_{\mu \mu}^{\ell}\right|$ and $\left|\lambda_{b s}^{q}\right|$ dictated by LFU in $\tau$ decays and $\Delta m_{B_{q}}$ mixing (see section 3.4). However, in the 95\% CL $(68 \% \mathrm{CL})$ preferred region of the model parameters the effective coupling $\left|\lambda_{\mu \mu}^{\ell}\right|$ can exceed $0.10(0.05)$. In this case $\Delta C_{9}^{\mu}$ can be within $1 \sigma$ or $2 \sigma$ of its central value (see right panels in figure 1$){ }^{7}$

In summary, we find that the effective Lagrangian in eq. (2.1) provides a significantly improved fit to low-energy data. It is worth stressing that, even from the EFT point of view, this model is highly constrained given the underlying set of dynamical hypotheses. As a result, the model leads to a series of predictions often different (more precise) than those obtained using more general EFT approaches (see e.g. [20, 21, 31]). The main predictions,

\footnotetext{
${ }^{7} \mathrm{~A}$ "perfect fit" of $\Delta C_{9}^{\mu}$ can be obtained extending the minimal version of the model, at the cost of introducing more free parameters. In particular, a natural extension is obtained with the inclusion of a $\mathrm{SU}(2)_{L}$ singlet, coupled to the current $J_{\mu}^{0}$ obtained by $J_{\mu}^{a}$ in eq. (2.2) with the replacement $T^{a} \rightarrow 1, g_{q \ell} \rightarrow g_{q \ell}^{\prime}$.
} 

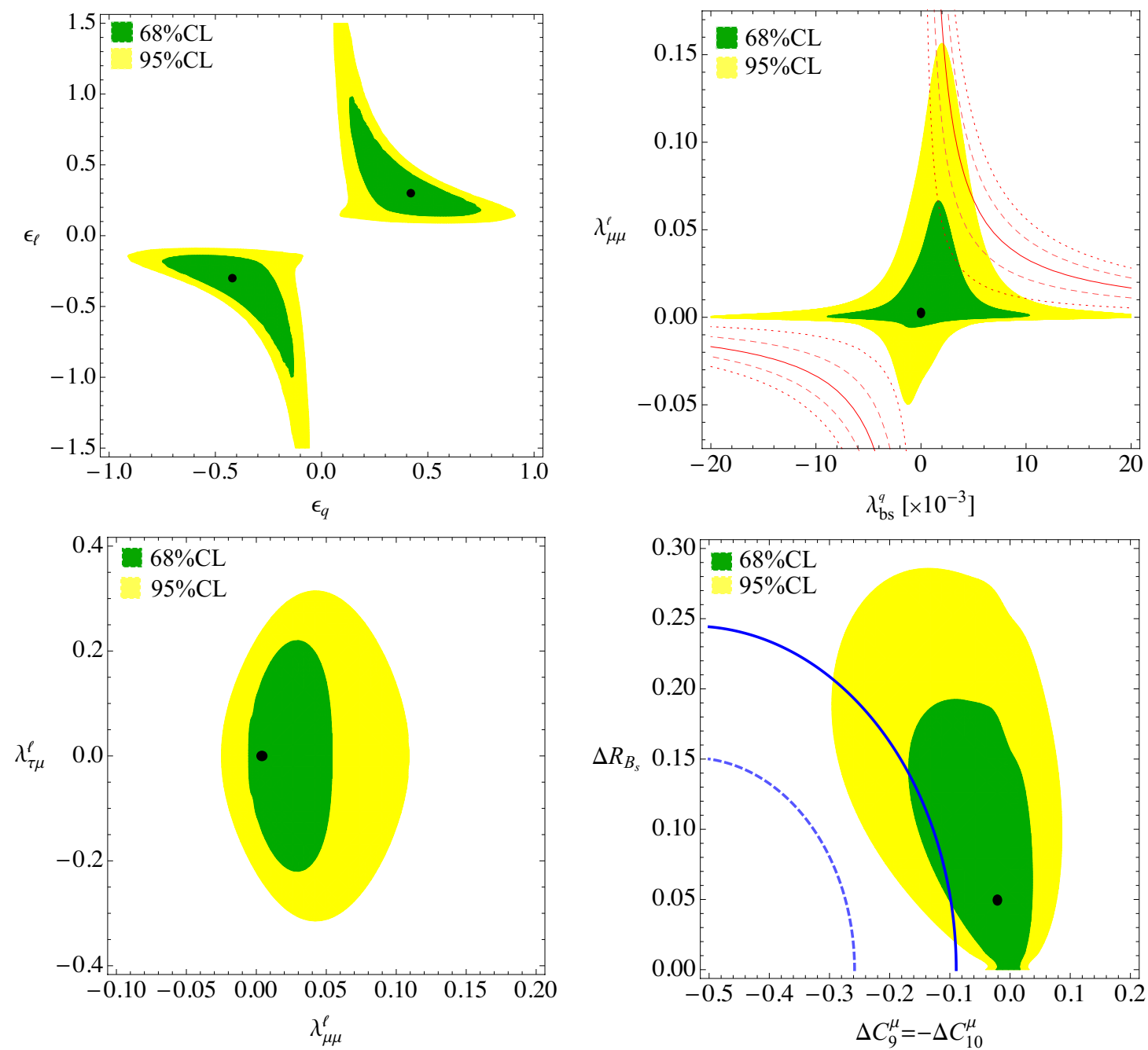

Figure 1. Results of the low-energy fit in table 1: 68\%CL (green) and 95\%CL (yellow) allowed regions in the $\left(\epsilon_{q}, \epsilon_{\ell}\right)$ plane (upper-left plot), $\left(\lambda_{b s}^{q}, \lambda_{\mu \mu}^{\ell}\right)$ plane (upper-right plot), $\left(\lambda_{\mu \mu}^{\ell}, \lambda_{\tau \mu}^{\ell}\right)$ plane (lower-left plot), and in the $\left(\Delta C_{9}^{\mu}, \Delta R_{B_{s}}\right)$ plane (lower-right plot), after having marginalised over the variables not shown. The black dots represent the best-fit points for these $2 \mathrm{~d}$ likelihoods. In the upper-right plot, the solid, dashed, and dotted red lines represent the iso-lines respectively for the best-fit, 1 - and 2- $\sigma$ ranges for $\Delta C_{9}^{\mu}$, with fixed $R_{0}=0.15$. In the lower-right plot, the dashed and solid blue lines represent the $68 \% \mathrm{CL}$ and $98 \% \mathrm{CL}$ regions for $\Delta C_{9}^{\mu}$ and $\Delta R_{B_{s}}$ as favored by $b \rightarrow s \mu^{+} \mu^{-}$and $\Delta m_{B_{s}}$ data.

which can be used to test the model in a more stringent way in view of future data, can be summarized as follows:

Charged currents. The $b \rightarrow c(u) \tau \nu$ charged currents should exhibit a universal enhancement (independent of the hadronic final state). This implies, in particular, $R_{B \tau \nu}=R_{D}^{\tau / \mu}=R_{D^{*}}^{\tau / \mu}$. LFU violations between $b \rightarrow c(u) \mu \nu$ and $b \rightarrow c(u) e \nu$ can be as large as $O(1 \%)$. The inclusive $\left|V_{c b}\right|$ and $\left|V_{u b}\right|$ determinations are enhanced over the exclusive ones because of the $\tau$ contamination in the corresponding samples. 
FCNC. The modification of the $b \rightarrow s \ell^{+} \ell^{-}$operators are purely left-handed. This implies, in particular, $\Delta C_{9}^{\mu}=-\Delta C_{10}^{\mu}$, hence a suppression (in the $10 \%-20 \%$ range) of $\mathcal{B}\left(B_{s, d} \rightarrow \mu^{+} \mu^{-}\right)$rates compared to their SM expectations. The central value of the anomaly in $R_{K}^{\mu / e}$ is likely to decrease (to $\sim 10 \%$ or less). The NP contribution to the $b \rightarrow s \tau^{+} \tau^{-}$amplitude is likely to be close, in magnitude, to the SM one. This implies a rate enhancement of at most a factor of $\approx 5$ compared to the SM (constructive interference) or a strong suppression (destructive interference). The magnitude of the FCNC-LFV transitions $b \rightarrow s \mu^{ \pm} \tau^{\mp}$ is at most $10 \%$ (in the rates) compared to the $b \rightarrow s \ell^{+} \ell^{-}$ones.

Meson-antimeson mixing. A $O(10 \%)$ deviation from the SM is expected $B_{s}$ mixing, if the anomaly in $R_{K}^{\mu / e}$ persists. According to the most plausible breaking structure of the $\mathrm{U}(2)_{q}$ symmetry, this deviation should be present also in $B_{d}$ mixing and should preserve the relation $\Delta M_{B_{s}} / \Delta M_{B_{d}}=\left(\Delta M_{B_{s}} / \Delta M_{B_{d}}\right)_{\mathrm{SM}}$. The $D-\bar{D}$ mixing amplitude should acquire a $\mathrm{CP}$-violating phase, whose magnitude could be just below the current experimental bounds.

$\tau$ decays. The $\tau \rightarrow 3 \mu$ and $\mu \rightarrow 3 e$ processes are generated at the tree level (contrary to LFV dipole transitions $\ell_{i} \rightarrow \ell_{j} \gamma$ ) and could be close to the present experimental bounds, although no precise correlations with other observables can be derived at present. If the anomaly in $R_{K}^{\mu / e}$ persists, violations of LFU in $\tau \rightarrow \mu \bar{\nu} \nu$ vs. $\tau \rightarrow e \bar{\nu} \nu$ are expected to be just below the current experimental bounds.

\section{Constraints on the dynamical model}

\subsection{Bounds from LEP-I}

Since the couplings of the heavy vector with SM fermions in this model are strongly non-universal, we cannot apply the LEP-I constraints as encoded in the bound on the $S$-parameter. Instead, we consider the non-universal fit of LEP-I data performed in the context of dimension- 6 operators in ref. [46]. To do this, we translate the effective Lagrangian of eq. (2.10) to the Higgs basis used for the fit.

Since the constraints on the $Z$ couplings to third generation (left-handed) quarks and leptons are of the same order as the bounds on the couplings to lighter fermions, while in our model the third generation is the one with biggest couplings, the strongest constraints on the model will arise from the bounds on $Z$ couplings to third generation fermions. This motivates us to simplify the analysis of the EFT fit by neglecting $\lambda_{i j}^{q, \ell}$ for $i, j \neq 3$. In this limit the fit only depends on these two combinations of parameters:

$$
\epsilon_{\ell} \epsilon_{H} \equiv \frac{g_{\ell} g_{H} m_{W}^{2}}{g^{2} m_{V}^{2}}=(4.3 \pm 8.7) \times 10^{-4}, \quad \epsilon_{q} \epsilon_{H} \equiv \frac{g_{q} g_{H} m_{W}^{2}}{g^{2} m_{V}^{2}}=(-0.8 \pm 1.4) \times 10^{-3},
$$

and the correlation is negligible. We introduced the adimensional parameters $\epsilon_{X} \equiv \frac{g_{X} m_{W}}{g m_{V}}$, with $X=\ell, q, H$. With this notation the constraint in eq. (3.4) from charged current $B$ decays can be written as $R_{0}=\epsilon_{\ell} \epsilon_{q}=0.14 \pm 0.04$. In figure 2 we combine these experimental 


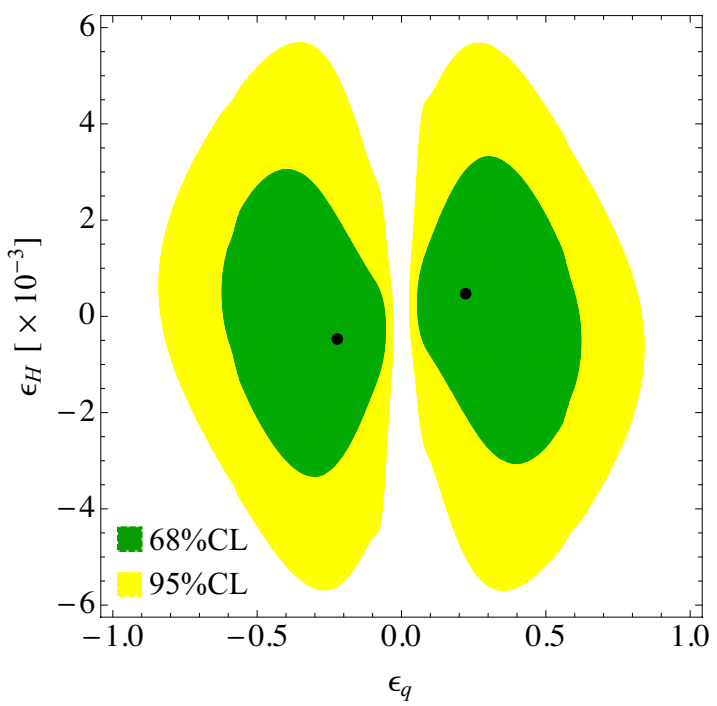

Figure 2. Results of the combined flavor and electroweak fit: 68\%CL (green) and 95\%CL (yellow) allowed regions in the $\left(\epsilon_{q}, \epsilon_{H}\right)$ plane after having marginalized over the other parameters. The black dots represent the best-fit points.

constraints with the ones from flavor physics and show the $68 \% \mathrm{CL}$ and $95 \% \mathrm{CL}$ allowed regions in the $\left(\epsilon_{q}, \epsilon_{H}\right)$ plane. From this we conclude that $\left|\epsilon_{H}\right| \lesssim 5 \times 10^{-3}$. This result allows us to conclude that, in absence of new degrees of freedom in the model, the massive vectors decay dominantly to SM fermions by means of the last term $\left(V_{\mu}^{a} J_{\mu}^{a}\right)$ in eq. (2.9).

\subsection{High-energy searches}

We parametrize massive vector boson couplings to SM fermions (in their mass-eigenstate basis) as follows

$$
\Delta \mathcal{L}_{V J}=V_{\mu}^{a} J_{\mu}^{a}=c_{i j}^{V} \bar{f}_{L}^{i} \gamma^{\mu} f_{L}^{j} V_{\mu} .
$$

With this definition, the two body $V \rightarrow \bar{f}_{i} f_{j}$ decay width is

$$
\Gamma\left(V \rightarrow \bar{f}_{i} f_{j}\right)=\frac{m_{V}}{24 \pi} N_{C}\left|c_{i j}^{V}\right|^{2} \mathcal{F}\left(\frac{m_{f_{i}}}{m_{V}}, \frac{m_{f_{j}}}{m_{V}}\right),
$$

where

$$
\mathcal{F}(x, y)=\left(1-\frac{x^{2}+y^{2}}{2}-\frac{\left(x^{2}-y^{2}\right)^{2}}{2}\right) \sqrt{1-2\left(x^{2}+y^{2}\right)-\left(x^{2}-y^{2}\right)^{2}},
$$

$N_{C}$ is the dimension of the color representation of the fermions, and we have assumed $m_{V}>m_{f_{i}}+m_{f_{j}}$.

Due to the approximate $\mathrm{U}(2)_{q} \times \mathrm{U}(2)_{\ell}$ symmetry, the total decay width of the vector bosons is dominated by decays to third-generation fermions. In the limit $m_{V}^{2} \gg 4 m_{t}^{2}$,

$$
\frac{\Gamma_{V^{ \pm}}}{m_{V^{ \pm}}} \approx \frac{\Gamma_{V^{0}}}{m_{V^{0}}} \approx \frac{1}{48 \pi}\left(g_{\ell}^{2}+3 g_{q}^{2}\right) .
$$

The neutral vector boson predominantly decays to $\tau^{+} \tau^{-}, \bar{\nu}_{\tau} \nu_{\tau}, \bar{b} b$, and $\bar{t} t$ final states. The relative impact of the leptonic and hadronic decay modes is driven by the ratio $g_{\ell} / g_{q}$ 

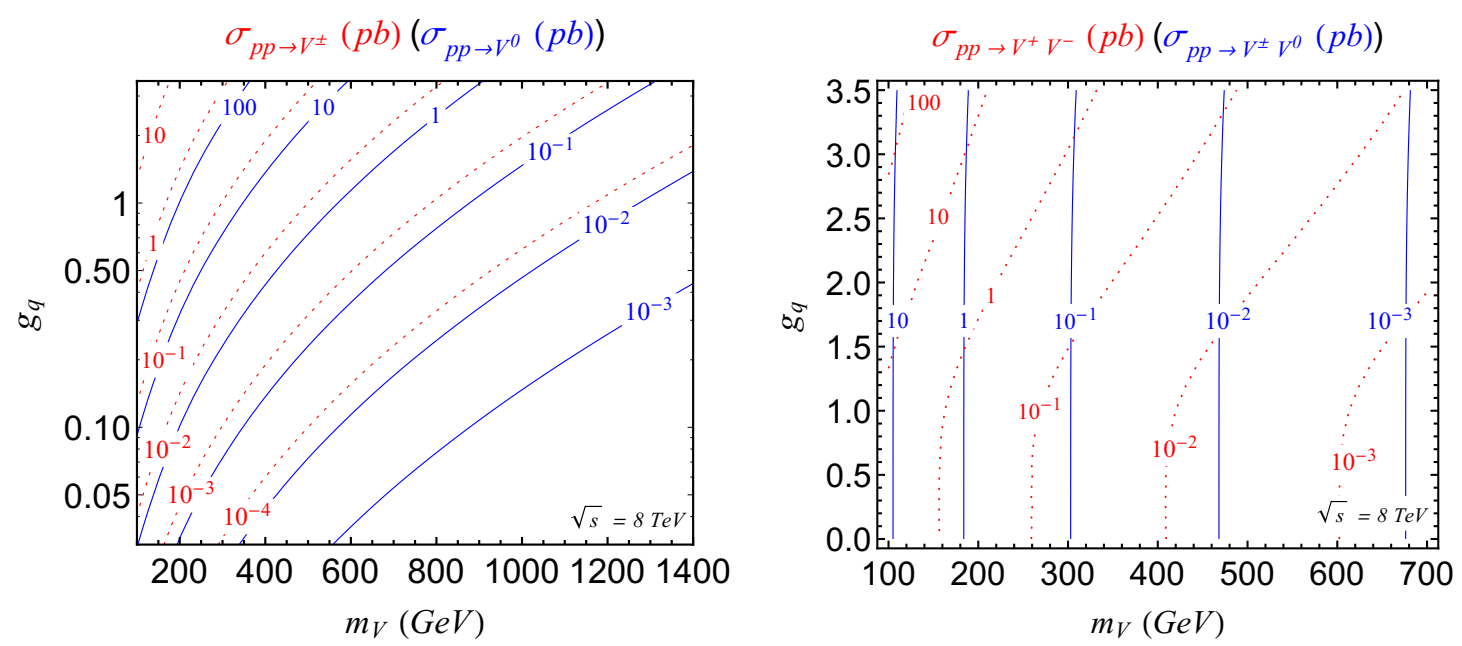

Figure 3. Predicted cross sections for single and pair production of charged and neutral vector bosons for $8 \mathrm{TeV} p p$ collisions as a function of $g_{q}$ and $m_{V}$ in the limit of approximate flavor symmetry.

(and the phase-space corrections to the large top-quark mass). The decay to a muon pair is parametrically suppressed by the smallness of $\lambda_{\mu \mu}^{\ell}$. In particular, the following relation holds

$$
\mathcal{B}\left(V^{0} \rightarrow \mu^{+} \mu^{-}\right)=\left|\lambda_{\mu \mu}^{\ell}\right|^{2} \mathcal{B}\left(V^{0} \rightarrow \tau^{+} \tau^{-}\right) .
$$

The dominant charged vector decay modes are $t \bar{b}$ and $\tau^{+} \nu_{\tau}$. In the following we assume $m_{V^{+}}>m_{t}$, such that the $V^{+}$state cannot be produced on-shell from top decays. We checked by explicit computation that when this criteria is satisfied the corrections to $t \rightarrow$ $b \tau^{+} \nu_{\tau}$ decay are well below present experimental sensitivity. The decays of both charged and neutral states to SM gauge bosons are strongly suppressed due to the strong limits on the $\epsilon_{H}$ parameter from electroweak precision data (see section 4.1).

The single vector bosons production at the LHC is dominated by Drell-Yan type processes, i.e. $p p \rightarrow V+X$, where $X$ stands for additional hadronic activity. While resonance searches in general impose severe limits on sequential (SM-like) $W^{\prime}$ and $Z^{\prime}$ bosons, we find significantly milder limits within our model. This is because of the specific flavor structure which suppresses both the production cross section and the decays into muon or electron final states.

In order to derive the present collider limits on the model, we have confronted the predictions of the model to a number of ATLAS and CMS searches for heavy $W^{\prime}$ and $Z^{\prime}$ resonances [47-53]. To this purpose, we have implemented the model in Universal FeynRules Output (UFO) [56] using the FeynRules [55] package version 2.3.1. We have used the MG5_aMC_v2.2.3 [57] package to simulate the tree-level $p p \rightarrow V^{ \pm}$and $p p \rightarrow$ $V^{0}$ production at $\sqrt{s}=8 \mathrm{TeV}$ in the 5 -flavor scheme. Finally, we have validated the implementation of the heavy vector triplet couplings to fermions by simulating decays and comparing the numerical results with the analytic expressions in eq. (4.3).

In figure 3 (left) we show the predicted cross sections (in pb) for $p p \rightarrow V^{ \pm}\left(p p \rightarrow V^{+}\right.$ plus $p p \rightarrow V^{-}$) and $p p \rightarrow V^{0}$, as obtained in the limit $\lambda_{i j}^{q}=\delta_{i 3} \delta_{3 j}$ (i.e. exact flavor symmetry, but for the breaking terms induced by the SM Yukawa couplings). In this limit 
the production cross sections are completely determined by $g_{q}$ and $m_{V}$. As can be seen, the neutral cross-section is about 100 times larger than the charged one. This is because the $V^{0}$ state is produced by bottom-bottom fusion, that is allowed in the limit of exact flavor symmetry, while the leading $V^{ \pm}$production channel is bottom-charm fusion, that is suppressed by $\left|V_{c b}\right|$ at the amplitude level.

The search for $W^{\prime} \rightarrow t \bar{b}$ with the ATLAS detector at $8 \mathrm{TeV}$ and $20.3 \mathrm{fb}^{-1}$ of data excludes a left-handed $W^{\prime}$ boson with a mass of $500(1000) \mathrm{GeV}$ if $\sigma\left(p p \rightarrow W^{\prime}\right) \times \mathcal{B}\left(W^{\prime} \rightarrow\right.$ $t \bar{b})>3.3(0.19) \mathrm{pb}[47]$. In addition, the CMS search for $W^{\prime} \rightarrow \tau \nu$ performed at $8 \mathrm{TeV}$ with an integrated luminosity of $19.7 \mathrm{fb}^{-1}$ excludes a $W^{\prime}$ boson with mass 300 (500) GeV if $\sigma\left(p p \rightarrow W^{\prime}\right) \times \mathcal{B}\left(W^{\prime} \rightarrow \tau \nu\right)>4(0.1)$ pb [48]. Comparing these limits with the predicted cross sections shown in figure 3 (left), we conclude that these searches have little impact on our model. We also checked that the ATLAS search for $W^{\prime} \rightarrow \mu \nu$ [49] has no relevance due to the limit on the $\left|\lambda_{\mu \mu}^{\ell}\right|$ coupling.

The resonance searches for neutral vector bosons are more relevant due to the larger expected cross section. The ATLAS search for $Z^{\prime}$ resonances decaying to $\tau^{+} \tau^{-}$using $19.5-20.3 \mathrm{fb}^{-1}$ of $8 \mathrm{TeV}$ data [50] sets an important constraint on the parameter space of the model. The exclusion limits (under the assumption of a narrow resonance) are shown in figure 4 in cyan solid (dashed) line assuming $\mathcal{B}\left(Z^{\prime} \rightarrow \tau^{+} \tau^{-}\right)=0.01(0.10)$. The region above these lines is excluded at $95 \% \mathrm{CL}$. The exclusion limits start from $m_{V}=500 \mathrm{GeV}$ because ref. [50] reports the limits on $\sigma \times \mathcal{B}$ only above this mass. To overcome this problem, we urge the experimental collaborations to extend the search for $Z^{\prime}$ resonances even in the low mass region. To extract the present limits for $m_{V}<500 \mathrm{GeV}$, we use the CMS search for the neutral MSSM Higgs boson decaying to a pair of tau leptons [54] at $8 \mathrm{TeV}$ and $19.7 \mathrm{fb}^{-1}$ luminosity. The collaboration reports a model independent limit on the $b \bar{b}$-induced production cross section times $\mathcal{B}\left(H \rightarrow \tau^{+} \tau^{-}\right)$(assuming a narrow resonance) in the region $100 \mathrm{GeV}<m_{H}<1 \mathrm{TeV}$. We have performed a parton-level MadGraph simulation to compare the kinematics of the $\tau^{+} \tau^{-}$final state produced by a scalar and a vector resonance (of mass $200 \mathrm{GeV}$ ). Having found small differences, we have re-interpreted the CMS bound into the $\sigma \times \mathcal{B}$ limit for our model reported in figure 4 . In particular, the region above pink solid (dashed) line is excluded assuming $\mathcal{B}\left(Z^{\prime} \rightarrow \tau^{+} \tau^{-}\right)=0.01(0.10)$. While the searches for dimuon resonances are usually more sensitive than the $\tau^{+} \tau^{-}$ones (see for instance [51]), we find them less relevant for our model due to smallness of $\lambda_{\mu \mu}^{\ell}$. On the other hand, dijet [52] and $t \bar{t}$ [53] resonance searches set limits on the cross section times branching ratio for $m_{V} \sim 1 \mathrm{TeV}$ of the order of $1 \mathrm{pb}$.

The impact of the direct searches in the $\tau^{+} \tau^{-}$channel on the parameter region preferred by flavor data is illustrated in figure 4 . The wide light green region is obtained imposing $R_{0}=0.14 \pm 0.04$ (68\% CL region) and $g_{q}, g_{\ell}<\sqrt{4 \pi}$. The narrower dark green (yellow) band is the region for which $R_{0}$ is within $68 \% \mathrm{CL}(95 \% \mathrm{CL})$ and $g_{q}=g_{\ell}$. In the minimal model, the predicted $V^{0} \rightarrow \tau^{+} \tau^{-}$branching ratio for $g_{q}=g_{\ell}$ is $\mathcal{B}\left(V^{0} \rightarrow \tau^{+} \tau^{-}\right) \approx 1 / 8$. Comparing with the exclusion curve obtained for $\mathcal{B}\left(Z^{\prime} \rightarrow \tau^{+} \tau^{-}\right)=0.1$ we deduce that the minimal model is ruled-out for $g_{q}=g_{\ell}$. The situation improves for $g_{\ell} \ll g_{q}$ (a configuration also preferred by flavor data, albeit with the lower bound $g_{\ell} \gtrsim g_{q} / 5.4$ from $D-\bar{D}$ mixing, see section 3.2), given $\mathcal{B}\left(V^{0} \rightarrow \tau^{+} \tau^{-}\right) \approx(1 / 8) \times\left(g_{\ell} / g_{q}\right)^{2}$. However, it is not possible to 


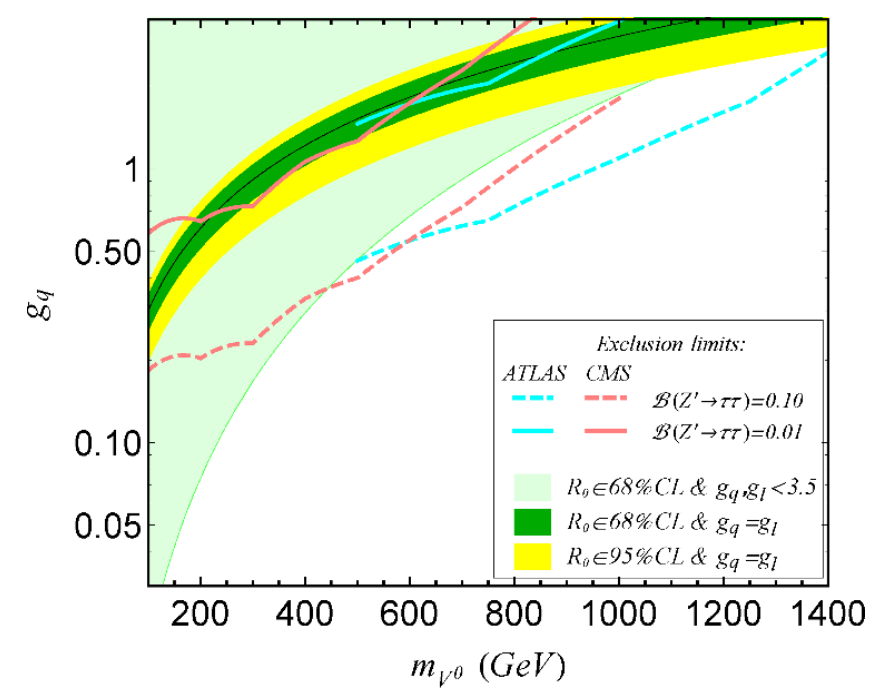

Figure 4. Preferred region from flavor data and exclusion limits from LHC. See text for details.

completely evade the bounds for perturbative values of the couplings $\left(g_{q}, g_{\ell}<\sqrt{4 \pi}\right)$ in the region preferred by flavor data.

There are different ways to evade the LHC limits on $p p \rightarrow V^{0} \rightarrow \tau^{+} \tau^{-}$going beyond the minimal model. The simplest possibility is to add new $V^{0}$ decay channels, say to a dark sector. This would result into lower values of $\mathcal{B}\left(V^{0} \rightarrow \tau^{+} \tau^{-}\right)$. As can be seen in figure 4 if, for $g_{q}=g_{\ell}, \mathcal{B}\left(V^{0} \rightarrow \tau^{+} \tau^{-}\right)$decreases to 0.01 , then there are regions of the parameter space that are allowed, both at low and at high masses. Another option is to consider a heavy $V^{0}$ in the limit of a strongly coupled theory $\left(\Gamma_{V} \sim M_{V}\right)$. In this case the resonance becomes broad and the limits obtained assuming a narrow state no longer holds. A third possibility would be to add an additional neutral heavy vector, e.g. a $\mathrm{SU}(2)_{L}$ singlet, close in mass to the neutral component of the triplet, with couplings tuned to interfere destructively with $V^{0}$ in the $p p \rightarrow \tau^{+} \tau^{-}+X$ cross section.

We finally comment about the $p p \rightarrow V V$ (pair production) process at the LHC. This proceeds via: i) $t$-channel quark-exchange diagrams, controlled by eq. (4.2), and ii) $s$ channel diagrams with off-shell SM electroweak gauge bosons. In the limit of no mixing with $\mathrm{EW}$ gauge bosons, $g_{H} \approx 0$, and neglecting contributions from additional non-minimal operators [45], the relevant interactions of the heavy vectors with the SM electroweak gauge bosons described by eq. (2.9) are

$$
\begin{aligned}
\mathcal{L} \supset & -i g\left(s_{\theta} A_{\mu}+c_{\theta} Z_{\mu}\right) V_{\nu}^{-}\left(\partial^{\mu} V^{+\nu}-\partial^{\nu} V^{+\mu}\right)-i g W^{+\mu} V^{0 \nu}\left(\partial_{\mu} V_{\nu}^{-}-\partial_{\nu} V_{\mu}^{-}\right) \\
& +i g W_{\mu}^{+} V_{\nu}^{-}\left(\partial^{\mu} V^{0 \nu}-\partial^{\nu} V^{0 \mu}\right)+\text { h.c. },
\end{aligned}
$$

where $g$ is the $\mathrm{SU}(2)_{L}$ coupling constant and $s_{\theta}\left(c_{\theta}\right)$ is sine (cosine) of the Weinberg angle. As for the single $V$ production, we have used MadGraph to simulate $p p \rightarrow V^{+} V^{-}$and $p p \rightarrow V^{ \pm} V^{0}$ (that is, $p p \rightarrow V^{+} V^{0}$ plus $p p \rightarrow V^{-} V^{0}$ ). In figure 3 (right) we show the predicted cross sections (in $p b$ ) for $8 \mathrm{TeV}$ proton-proton collisions, as obtained in the limit $\lambda_{i j}^{q}=\delta_{i 3} \delta_{3 j}$. As illustrated with the vertical iso-lines, the cross sections are dominated by 
$s$-channel diagrams for small $g_{q}$ couplings. On the other hand, for large couplings there is a substantial contribution from the $b \bar{b}$ induced $t$-channel diagram to $V^{+} V^{-}$production. Based on the predicted cross sections, we conclude that the single production is more relevant compared to the pair production for the interesting region of the parameter space.

\section{Conclusions}

Lepton Flavor Universality is not a fundamental symmetry: within the Standard Model it is an approximate accidental symmetry broken only by the Yukawa interactions. This specific symmetry and symmetry-breaking pattern results in tiny deviations from LFU in helicity-conserving amplitudes, within the SM, and it implies that LFU tests are clean probes of physics beyond the SM.

Motivated by a series of recent experimental results in $B$ physics pointing to possible violations of LFU, both in charged and in neutral currents, in this paper we have consider a simplified dynamical model able to describe these effects in a unified way. In particular, we have shown that a $\mathrm{SU}(2)_{L}$ triplet of massive vector bosons, coupled predominantly to third generation fermions (both quarks and leptons), can significantly improve the description of present data.

The proposed model has a series of virtues compared to previous attempts to describe such effects in terms of New Physics: i) it connects the breaking of LFU between charged and neutral currents, and between semi-leptonic and purely leptonic processes; ii) it is based on a simple flavor symmetry, whose breaking terms are related to the structure of the SM Yukawa couplings, both in the quark and in the lepton sector; iii) it connects low-energy deviations from the SM to direct searches for NP at high $p_{T}$. The constrained structure of the model makes it highly non trivial to satisfy all existing bounds and, at the same time, accommodate deviations from the SM as large as indicated by the central values in eqs. (1.1)-(1.3). We find that this happens quite naturally in the case of charged currents, both at low and at high energies. The situation is more problematic in the case of neutral currents. On the one hand, the maximal deviations from unity in $R_{K}^{\mu / e}$ barely exceed $10 \%$. On the other hand, the minimal version of the model is ruled out by the direct searches for resonances decaying into $\tau^{+} \tau^{-}$at ATLAS and CMS. As discussed, both these issues can be improved with less minimal versions of the model, at the cost of introducing more free parameters.

One of the most remarkable aspects of the minimal version of the model is the welldefined pattern of deviations in low-energy processes listed at the end of section 3.5. This pattern is largely insensitive to possible extensions of the model necessary to overcome the constraints from direct searches. It mainly reflects the symmetry structure of the model and could be used, in the near future, to verify or falsify this framework with more precise data.

Besides the specific predictions of the proposed model, we stress the importance of future experimental tests of LFU at low-energies, and dedicated searches for flavor-nonuniversal phenomena at high energies. On the low-energy side, we urge the experimental collaborations to re-analyze charged-current $B$ decays without assuming lepton flavor universality. On the high-energy side, we encourage the search for deviations from the SM 
in $\tau^{+} \tau^{-}$and $t \bar{t}$ invariant-mass distributions, relaxing the hypothesis of narrow resonances and covering also the region of low invariant masses.

\section{Acknowledgments}

We thank Riccardo Barbieri, Andreas Crivellin, Martin Schmaltz, and Nicola Serra for useful comments and discussions. This research was supported in part by the Swiss National Science Foundation (SNF) under contract 200021-159720.

Open Access. This article is distributed under the terms of the Creative Commons Attribution License (CC-BY 4.0), which permits any use, distribution and reproduction in any medium, provided the original author(s) and source are credited.

\section{References}

[1] BABAR collaboration, J.P. Lees et al., Measurement of an excess of $\bar{B} \rightarrow D^{(*)} \tau^{-} \bar{\nu}_{\tau}$ decays and implications for charged Higgs bosons, Phys. Rev. D 88 (2013) 072012 [arXiv: 1303.0571] [INSPIRE].

[2] T. Kuhr, New $B \rightarrow D^{(*)} \tau \nu$ result from Belle, talk presented at Flavor Physics \& $C P$ violation (FPCP2015), May 25-29, Nagoya, Japan (2015).

[3] G. Ciezarek, New $D^{(*)} \tau \nu$ result from $L H C b+$ non-B semileptonics, talk presented at Flavor Physics 83 CP violation (FPCP2015), May 25-29, Nagoya, Japan (2015).

[4] LHCb collaboration, Test of lepton universality using $B^{+} \rightarrow K^{+} \ell^{+} \ell^{-}$decays, Phys. Rev. Lett. 113 (2014) 151601 [arXiv: 1406.6482] [INSPIRE].

[5] Particle Data Group collaboration K.A. Olive et al., Review of particle physics, Chin. Phys. C 38 (2014) 090001 [inSPIRE].

[6] S. Fajfer, J.F. Kamenik and I. Nisandzic, On the $B \rightarrow D^{*} \tau \bar{\nu}_{-} \tau$ sensitivity to new physics, Phys. Rev. D 85 (2012) 094025 [arXiv: 1203.2654] [inSPIRE].

[7] D. Becirevic, N. Kosnik and A. Tayduganov, $\bar{B} \rightarrow D \tau \bar{\nu}_{-} \tau$ vs. $\bar{B} \rightarrow D \mu \bar{\nu}_{-} \mu$, Phys. Lett. B 716 (2012) 208 [arXiv: 1206. 4977] [INSPIRE].

[8] G. Hiller and F. Krüger, More model independent analysis of $b \rightarrow s$ processes, Phys. Rev. D 69 (2004) 074020 [hep-ph/0310219] [INSPIRE].

[9] LHCb collaboration, Measurement of form-factor-independent observables in the decay $B^{0} \rightarrow K^{* 0} \mu^{+} \mu^{-}$, Phys. Rev. Lett. 111 (2013) 191801 [arXiv: 1308.1707] [INSPIRE].

[10] C. Langenbruch, Latest results on rare decays from LHCb, arXiv:1505.04160.

[11] S. Descotes-Genon, T. Hurth, J. Matias and J. Virto, Optimizing the basis of $B \rightarrow K^{*} l l$ observables in the full kinematic range, JHEP 05 (2013) 137 [arXiv:1303.5794] [INSPIRE].

[12] Heavy Flavor Averaging Group collaboration, Y. Amhis et al., Averages of b-hadron, c-hadron and $\tau$-lepton properties as of summer 2014, arXiv:1412.7515 [INSPIRE].

[13] S. Fajfer, J.F. Kamenik, I. Nisandzic and J. Zupan, Implications of lepton flavor universality violations in B decays, Phys. Rev. Lett. 109 (2012) 161801 [arXiv:1206.1872] [INSPIRE].

[14] S. Descotes-Genon, J. Matias and J. Virto, Understanding the $B \rightarrow K^{*} \mu^{+} \mu^{-}$anomaly, Phys. Rev. D 88 (2013) 074002 [arXiv: 1307.5683] [INSPIRE]. 
[15] W. Altmannshofer and D.M. Straub, New physics in $B \rightarrow K^{*} \mu \mu$ ?, Eur. Phys. J. C 73 (2013) 2646 [arXiv: 1308.1501] [inSPIRE].

[16] A. Datta, M. Duraisamy and D. Ghosh, Explaining the $B \rightarrow K^{*} \mu^{+} \mu^{-}$data with scalar interactions, Phys. Rev. D 89 (2014) 071501 [arXiv:1310.1937] [InSPIRE].

[17] G. Hiller and M. Schmaltz, $R_{K}$ and future $b \rightarrow$ sll physics beyond the standard model opportunities, Phys. Rev. D 90 (2014) 054014 [arXiv: 1408.1627] [InSPIRE].

[18] G. Hiller and M. Schmaltz, Diagnosing lepton-nonuniversality in $b \rightarrow$ sll, JHEP 02 (2015) 055 [arXiv: 1411.4773] [INSPIRE].

[19] A. Crivellin and S. Pokorski, Can the differences in the determinations of $V_{u b}$ and $V_{c b}$ be explained by new physics?, Phys. Rev. Lett. 114 (2015) 011802 [arXiv:1407.1320] [INSPIRE].

[20] S.L. Glashow, D. Guadagnoli and K. Lane, Lepton flavor violation in B decays?, Phys. Rev. Lett. 114 (2015) 091801 [arXiv: 1411.0565] [INSPIRE].

[21] B. Bhattacharya, A. Datta, D. London and S. Shivashankara, Simultaneous explanation of the $R_{K}$ and $R\left(D^{(*)}\right)$ puzzles, Phys. Lett. B 742 (2015) 370 [arXiv:1412.7164] [INSPIRE].

[22] B. Gripaios, M. Nardecchia and S.A. Renner, Composite leptoquarks and anomalies in B-meson decays, JHEP 05 (2015) 006 [arXiv:1412.1791] [INSPIRE].

[23] D. Ghosh, M. Nardecchia and S.A. Renner, Hint of lepton flavour non-universality in $B$ meson decays, JHEP 12 (2014) 131 [arXiv:1408.4097] [INSPIRE].

[24] A. Crivellin, G. D'Ambrosio and J. Heeck, Explaining $h \rightarrow \mu^{ \pm} \tau^{\mp}, B \rightarrow K^{*} \mu^{+} \mu^{-}$and $B \rightarrow K \mu^{+} \mu^{-} / B \rightarrow K e^{+} e^{-}$in a two-Higgs-doublet model with gauged $L_{-} \mu-L_{-} \tau$, Phys. Rev. Lett. 114 (2015) 151801 [arXiv:1501.00993] [INSPIRE].

[25] A. Crivellin, G. D'Ambrosio and J. Heeck, Addressing the LHC flavor anomalies with horizontal gauge symmetries, Phys. Rev. D 91 (2015) 075006 [arXiv:1503.03477] [INSPIRE].

[26] D. Aristizabal Sierra, F. Staub and A. Vicente, Shedding light on the $b \rightarrow s$ anomalies with $a$ dark sector, Phys. Rev. D 92 (2015) 015001 [arXiv:1503.06077] [INSPIRE].

[27] D. Bečirević, S. Fajfer and N. Košnik, Lepton flavor nonuniversality in $b \rightarrow s \ell^{+} \ell^{-}$processes, Phys. Rev. D 92 (2015) 014016 [arXiv: 1503.09024] [INSPIRE].

[28] I. de Medeiros Varzielas and G. Hiller, Clues for flavor from rare lepton and quark decays, JHEP 06 (2015) 072 [arXiv: 1503.01084] [INSPIRE].

[29] A. Crivellin, L. Hofer, J. Matias, U. Nierste, S. Pokorski and J. Rosiek, Lepton-flavour violating $B$ decays in generic $Z^{\prime}$ models, arXiv:1504.07928 [INSPIRE].

[30] A. Celis, J. Fuentes-Martin, M. Jung and H. Serodio, Family non-universal $Z^{\prime}$ models with protected flavor-changing interactions, Phys. Rev. D 92 (2015) 1 [arXiv:1505.03079] [INSPIRE].

[31] R. Alonso, B. Grinstein and J.M. Camalich, Lepton universality violation and lepton flavor conservation in B-meson decays, arXiv:1505.05164 [INSPIRE].

[32] R. Barbieri, G. Isidori, J. Jones-Perez, P. Lodone and D.M. Straub, U(2) and minimal flavour violation in supersymmetry, Eur. Phys. J. C 71 (2011) 1725 [arXiv:1105. 2296] [InSPIRE].

[33] G. Blankenburg, G. Isidori and J. Jones-Perez, Neutrino masses and LFV from minimal breaking of $\mathrm{U}(3)^{5}$ and $\mathrm{U}(2)^{5}$ flavor symmetries, Eur. Phys. J. C 72 (2012) 2126 [arXiv: 1204.0688] [INSPIRE]. 
[34] Flavianet Working Group on Kaon Decays collaboration, M. Antonelli et al., An evaluation of $\left|V_{u} s\right|$ and precise tests of the standard model from world data on leptonic and semileptonic kaon decays, Eur. Phys. J. C 69 (2010) 399 [arXiv:1005.2323] [InSPIRE].

[35] LHCb collaboration, Determination of the quark coupling strength $\left|V \_u b\right|$ using baryonic decays, arXiv:1504.01568 [INSPIRE].

[36] R. Barbieri, D. Buttazzo, F. Sala and D.M. Straub, Flavour physics and flavour symmetries after the first LHC phase, JHEP 05 (2014) 105 [arXiv: 1402.6677] [INSPIRE].

[37] G. Buchalla, A.J. Buras and M.E. Lautenbacher, Weak decays beyond leading logarithms, Rev. Mod. Phys. 68 (1996) 1125 [hep-ph/9512380] [InSPIRE].

[38] G. Isidori, Flavor physics and CP-violation, arXiv:1302.0661 [INSPIRE].

[39] B. Stugu, Summary on tau leptonic branching ratios and universality, Nucl. Phys. Proc. Suppl. 76 (1999) 123 [hep-ex/9811048] [INSPIRE].

[40] W. Altmannshofer, S. Gori, M. Pospelov and I. Yavin, Neutrino trident production: a powerful probe of new physics with neutrino beams, Phys. Rev. Lett. 113 (2014) 091801 [arXiv: 1406.2332] [INSPIRE].

[41] CHARM-II collaboration, D. Geiregat et al., First observation of neutrino trident production, Phys. Lett. B 245 (1990) 271 [INSPIRE].

[42] CCFR collaboration, S.R. Mishra et al., Neutrino tridents and W Z interference, Phys. Rev. Lett. 66 (1991) 3117 [INSPIRE].

[43] W. Altmannshofer and D.M. Straub, New physics in $b \rightarrow s$ transitions after LHC run 1, arXiv: 1411.3161 [INSPIRE].

[44] W. Altmannshofer and D.M. Straub, Implications of $b \rightarrow s$ measurements, arXiv: 1503.06199.

[45] D. Pappadopulo, A. Thamm, R. Torre and A. Wulzer, Heavy vector triplets: bridging theory and data, JHEP 09 (2014) 060 [arXiv:1402.4431] [InSPIRE].

[46] A. Efrati, A. Falkowski and Y. Soreq, Electroweak constraints on flavorful effective theories, JHEP 07 (2015) 018 [arXiv: 1503.07872] [INSPIRE].

[47] ATLAS collaboration, Search for $W^{\prime} \rightarrow t \bar{b}$ in the lepton plus jets final state in proton-proton collisions at a centre-of-mass energy of $\sqrt{s}=8 \mathrm{TeV}$ with the ATLAS detector, Phys. Lett. B 743 (2015) 235 [arXiv: 1410.4103] [INSPIRE].

[48] CMS collaboration, Search for new physics in final states with a tau and missing transverse energy using pp collisions at $\sqrt{s}=8 \mathrm{TeV}$, CMS-PAS-EXO-12-011 (2012).

[49] ATLAS collaboration, Search for new particles in events with one lepton and missing transverse momentum in pp collisions at $\sqrt{s}=8 \mathrm{TeV}$ with the ATLAS detector, JHEP 09 (2014) 037 [arXiv: 1407.7494] [INSPIRE].

[50] ATLAS collaboration, A search for high-mass resonances decaying to $\tau^{+} \tau^{-}$in pp collisions at $\sqrt{s}=8 \mathrm{TeV}$ with the ATLAS detector, arXiv:1502.07177 [INSPIRE].

[51] ATLAS collaboration, Search for high-mass dilepton resonances in pp collisions at $\sqrt{s}=8$ TeV with the ATLAS detector, Phys. Rev. D 90 (2014) 052005 [arXiv:1405.4123] [INSPIRE].

[52] CMS collaboration, Search for resonances and quantum black holes using dijet mass spectra in proton-proton collisions at $\sqrt{s}=8 \mathrm{TeV}$, Phys. Rev. D 91 (2015) 052009 [arXiv: 1501.04198] [INSPIRE]. 


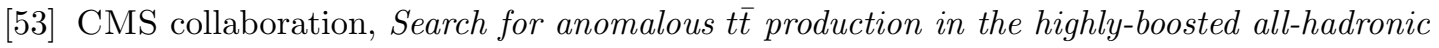
final state, JHEP 09 (2012) 029 [Erratum ibid. 1403 (2014) 132] [arXiv:1204.2488] [INSPIRE].

[54] CMS collaboration, Search for neutral MSSM Higgs bosons decaying to a pair of tau leptons in pp collisions, JHEP 10 (2014) 160 [arXiv:1408.3316] [INSPIRE].

[55] N.D. Christensen and C. Duhr, FeynRules - Feynman rules made easy, Comput. Phys. Commun. 180 (2009) 1614 [arXiv:0806.4194] [INSPIRE].

[56] C. Degrande, C. Duhr, B. Fuks, D. Grellscheid, O. Mattelaer and T. Reiter, UFO - The Universal FeynRules Output, Comput. Phys. Commun. 183 (2012) 1201 [arXiv:1108.2040] [INSPIRE].

[57] J. Alwall et al., The automated computation of tree-level and next-to-leading order differential cross sections and their matching to parton shower simulations, JHEP 07 (2014) 079 [arXiv: 1405.0301] [INSPIRE]. 